\title{
Deutsche Europawahlen künftig ohne Sperrklausel? Das Urteil des Bundesverfassungsgerichts vom November 2011 und seine Folgen
}

\author{
Rudolf Hrbek*
}

Das mit Blick auf die für 1979 vorgesehenen ersten Direktwahlen zum Europäischen Parlament im Sommer 1978 beschlossene Gesetz über die Wahl der Abgeordneten aus der Bundesrepublik Deutschland - das Europawahlgesetz $(E u W G)^{1}-$ enthielt in $\S 2$ Abs. 6 eine Fünfprozentsperrklausel. Die zwei gegen diese Bestimmung beim Bundesverfassungsgericht erhobenen Verfassungsbeschwerden wurden vom Zweiten Senat des Gerichts in einer einstimmig ergangenen Entscheidung vom 22. Mai $1979^{2}$ zurückgewiesen. Die von 1979 bis 2009 insgesamt sieben deutschen Europawahlen wurden dementsprechend bei Geltung einer Fünfprozentsperrklausel abgehalten.

Die Auswirkungen der Sperrklausel auf die an den Wahlen seit 1979 teilnehmenden Parteien lassen sich wie folgt zusammenfassen: ${ }^{3}$ auf die Grünen entfielen 1979 3,2 Prozent der Stimmen; die FDP scheiterte 1984 (4,8 Prozent), 1994 (4,1 Prozent) und 1999 (3,0 Prozent); auf die PDS entfielen 1994 4,7 Prozent; die Republikaner (1989 mit 7,1 Prozent erfolgreich) scheiterten 1994 (3,9 Prozent), 1999 (1,7 Prozent), 2004 (1,9 Prozent) und 2009 (1,3 Prozent); andere Parteien beziehungsweise Wählervereinigungen/Listen blieben, wenn sie kandidierten, ausnahmslos bei deutlich unter 2 Prozent, vielfach bei Werten von etwas über oder unter 1 Prozent, sodass auf sie - ohne Sperrklausel - lediglich ein, höchstens zwei Mandate entfallen wären. ${ }^{4}$

Im Anschluss an die Europawahlen 2009 war das Bundesverfassungsgericht erneut wegen der Frage der Zulässigkeit - also der Verfassungsmäßigkeit - der Fünfprozentsperrklausel (jetzt in $\S 2$ Abs. 7 EuWG enthalten) angerufen worden. In seinem am 9. November 2011 ergangenen Urteil ${ }^{5}$ hat der Zweite Senat des Gerichts entschieden, dass die Sperrklausel „,mit Artikel 3 Absatz 1 und Artikel 21 Absatz 1 des Grundgesetzes unvereinbar und daher nich-

* Prof. Dr. Rudolf Hrbek, Institut für Politikwissenschaft und Europäisches Zentrum für Föderalismus-Forschung, Universität Tübingen.

1 Gesetz über die Wahl der Abgeordneten des Europäischen Parlaments aus der Bundesrepublik Deutschland (Europawahlgesetz-EuWG) vom 16. Juni 1978, in: Bundesgesetzblatt Teil I, Nr. 31 vom 21. Juni 1978, S. 709-717.

2 BVerfGE 51, 222ff.

3 Dabei ist zu beachten, dass bei den ersten drei Europawahlen (1979, 1984 und 1989) das auf Deutschland entfallende Kontingent jeweils 78 Abgeordnete umfasste, bei den folgenden Wahlen (1994, 1999, 2004 und 2009) jeweils 99 Abgeordnete.

4 Dabei handelt es sich um die Friedensliste (1984 mit 1,3 Prozent), die DVU (1989 mit 1,6 Prozent), der Bund freier Bürger (1994 mit 1,1 Prozent), die Grauen (1994 mit 0,8 Prozent und 2004 mit 1,2 Prozent), die ÖDP (1994 mit 0,8 Prozent), die Tierschutz Partei (2004 mit 1,3 Prozent und 2009 mit 1,1 Prozent), die Familien Partei (2004 mit 1,0 Prozent und 2009 mit 1,0 Prozent), die NPD (2004 mit 0,9 Prozent), die Freien Wähler (2009 mit 1,7 Prozent) und die Piraten (2009 mit 0,9 Prozent). Gruppierungen mit noch geringeren Anteilen, die deshalb ohne Chancen auf ein Mandat waren, werden hier nicht aufgeführt.

5 BVerfGE 129, $130 \mathrm{ff}$. 
tig ${ }^{\circ 6}$ sei. Die Entscheidung war mit 5 gegen 3 Stimmen ergangen, ${ }^{7}$ wobei das Ergebnis von einem Mitglied des Senats ,,aus abweichenden Gründen“ mitgetragen wurde. ${ }^{8}$ Zwei Richter haben zum Urteil gemeinsam ihre „Abweichende Meinung ${ }^{69}$ - sie ist Teil des Urteils formuliert.

Der Gesetzgeber war damit gehalten, das EuWG mit Blick auf die nächsten Europawahlen 2014 zu ändern. Das erfolgte mit Beschluss des Bundestages vom 13. Juni 2013 über das Fünfte Gesetz zur Änderung des Europawahlgesetzes; ${ }^{10} \S 2$ Abs. 7 EuWG sieht jetzt eine Dreiprozentsperrklausel vor. Auch dagegen wurden bald nach Verkündigung und Inkrafttreten des Gesetzes beim Bundesverfassungsgericht Verfassungsklagen eingereicht und es ist zu erwarten, dass das Gericht rechtzeitig vor den nächsten im Mai 2014 stattfindenden Europawahlen über die Verfassungsmäßigkeit dieser jetzt niedrigeren Sperrklausel beschließen wird.

Es ist das Ziel dieses Beitrags, die kontroverse Diskussion über die Sperrklausel seit 1978 kurz nachzuzeichnen und die dabei vertretenen Auffassungen zu präsentieren und zu erörtern.

Der Beitrag resümiert einleitend generelle Wahlrechtsgrundsätze, insbesondere Kriterien zugunsten von Sperrklauseln. Er behandelt sodann die beiden Urteile des Verfassungsgerichts (1979 und 2011), mit Schwerpunkt auf dem Urteil von 2011. Danach wendet er sich der Reaktion des Gesetzgebers vom Sommer 2013 zu, bevor ein kurzer Ausblick den Beitrag abschließt.

\section{(Wahlrechts-)Grundsätze, die sich auf Einführung und Geltung von Sperrklauseln beziehen}

Zu den Merkmalen demokratischer Verfassungsstaaten gehören Wahlrechtsgrundsätze, wie sie beispielsweise in Art. 38 Abs. 1 des deutschen Grundgesetzes verankert sind. Danach werden die Abgeordneten des Deutschen Bundestages, ,in allgemeiner, unmittelbarer, freier, gleicher und geheimer Wahl gewählt.“"Von zentraler Bedeutung für die hier zu behandelnde Frage ist der Grundsatz der Gleichheit, wonach jede abgegebene Stimme den gleichen Zählwert hat. Davon zu unterscheiden ist der Erfolgswert der Wählerstimme, der vom Zählwert abweichen kann. Hier wirkt sich zum einen das Wahlsystem aus: Bei einem Mehrheitswahlsystem ,zählen` letztlich nur die für den Wahlkreisgewinner abgegebenen Stimmen. Ergänzend wirkt sich die Einteilung der Wahlkreise oder Wahlbezirke dergestalt aus, dass die Stimme des einzelnen Wählers bei einer geringeren Zahl von Wahlberechtigten ein relativ größeres Gewicht hat. Deshalb wird darauf geachtet, dass die durchschnittliche Zahl der Wahlberechtigten pro Wahlkreis innerhalb eines nicht zu breiten Korridors liegt. Auch im

6 Ebenda.

7 Christoph Schönberger spricht in diesem Zusammenhang von einem ,zerrissenen Senat“, dessen Urteil ,,innerhalb des Zweiten Senats nur die geringstmögliche Überzeugungskraft entfaltet“ habe. Vgl. Christoph Schönberger: Das Bundesverfassungsgericht und die Fünf-Prozent-Klausel bei der Wahl zum Europäischen Parlament, in: JuristenZeitung 2/2012, S. 80-86, hier S. 80-81.

8 Schönberger nennt dies ,,eigenartig“, denn „,[d]er fünfte Richter, mit dessen Stimme die Nichtigerklärung hier steht und fällt, lässt die Öffentlichkeit zwar wissen, dass ihn die Begründung seiner vier Senatskollegen nicht überzeugt, enthält dieser aber zugleich die Gründe vor, aus denen er selbst die Kassation der Sperrklausel gleichwohl mitträgt." Vgl. Schönberger: Das Bundesverfassungsgericht und die Fünf-Prozent-Klausel, 2012, S. 81 .

9 BVerfGE 129, 130, Rn. 147-160.

10 Fünftes Gesetz zur Änderung des Europawahlgesetzes vom 7. Oktober 2013, in: Bundesgesetzblatt Teil I, Nr. 60 vom 9. Oktober 2013, S. 3749-3751. 
Fall eines Verhältniswahlsystems, welches gegenwärtig in allen EU-Mitgliedstaaten für die Europawahlen gilt, spielt die Größe von Wahlbezirken eine Rolle, wenn es nämlich keine nationalen Listen gibt, sondern das gesamte Wahlgebiet in mehrere (in aller Regel unterschiedlich große) Wahlbezirke eingeteilt ist, in denen jeweils mehrere Mandate zu verteilen sind.

Vor allem beeinflusst aber bei Geltung des Verhältniswahlsystems eine Sperrklausel den Erfolgswert; Stimmen zugunsten einer Partei, die unterhalb der Sperrklausel-Grenze bleibt, sind gleichsam verloren. Weil der Grundsatz der Wahlrechtsgleichheit in einem demokratischen System ein hohes Gut ist, darf eine Sperrklausel nur aus zwingenden Gründen - und auch dann nur in bestimmter Höhe (eine international vergleichende Übersicht ergibt, dass die Marke von 5 Prozent dabei die obere Grenze darstellt) - eingeführt werden. Als solche Gründe gelten insbesondere die Gewährleistung der Funktionsfähigkeit des zu wählenden Parlaments (bezogen auf die Bildung einer handlungsfähigen Regierung und ihre verlässliche Unterstützung durch möglichst stabile Mehrheiten) und die Verhinderung einer zu großen Parteienzersplitterung.

In diesem Zusammenhang ist auch der Grundsatz der Chancengleichheit politischer Parteien zu beachten. Sperrklauseln mindern bei einem Verhältniswahlsystem die Chancen kleiner Parteien, Mandate zu gewinnen und im Parlament vertreten zu sein. Sie mindern auch die Chancen neu gegründeter Parteien, sich durch Präsenz im Parlament zu profilieren, dabei zu wachsen und sich im Parteiensystem zu etablieren. Hier kommt ein weiterer Faktor ins Spiel: Wähler könnten bei Geltung einer Sperrklausel bewusst darauf verzichten, eine kleinere oder neue Partei, die von der Sperrklausel ,bedroht' wird, zu wählen, um zu vermeiden, dass ihre Stimme ,verloren“ ist, also keinen Erfolgswert hat. Auch mit Blick auf den Grundsatz der Chancengleichheit politischer Parteien bedarf die Einführung von Sperrklauseln also besonderer Gründe.

\section{Die Entscheidung des Bundesverfassungsgerichts vom Mai 1979 zugunsten einer Fünfprozentsperrklausel im deutschen Europawahlgesetz ${ }^{11}$}

Mit seinem Beschluss vom 22. Mai 1979 hatte der Zweite Senat des Gerichts zwei Verfassungsbeschwerden gegen die in $\S 2$ Abs. 6 EuWG festgelegte Fünfprozentsperrklausel zurückgewiesen. Bei den Beschwerdeführern handelte es sich um einen wahlberechtigten deutschen Staatsbürger sowie die „Liga für Freie Völker - Europa 2000“ als eine mitgliedschaftlich organisierte politische Vereinigung, die sich mit einem eigenen Wahlvorschlag an der Europawahl 1979 beteiligen wollte.

Der klagende Bürger begründete seine Beschwerde wie folgt: Die Sperrklausel verletze sein Grundrecht auf Wahlrechtsgleichheit; von einer Parteienvielfalt im Europäischen Parlament sei keine wesentliche Beeinträchtigung der Funktionen der Versammlung zu befürchten; die Sperrklausel sei auch völlig ungeeignet, eine Parteienzersplitterung im Europäischen Parlament zu verhindern; die Klausel lasse sich auch nicht mit dem angeblichen Postulat nach nationaler Repräsentanz begründen; schließlich spräche auch der Vergleich mit anderen Mitgliedstaaten (nur in Frankreich gelte eine Fünfprozentsperrklausel) für die Beschwerde. Die „Liga für Freie Völker“ machte geltend, dass die Sperrklausel den Erfolgswert der Stimmen der von ihr betroffenen Gruppen mindere und dass sich die Klausel

11 Die Ausführungen in diesem Abschnitt folgen dem Text des Beschlusses des Zweitens Senats. Die entsprechenden Textstellen werden durchgehend in indirekter Rede wiedergegeben und nicht als wörtliche Zitate. 
allein durch die Möglichkeit ihres Wirksamwerdens negativ bei potenziellen Wählern auswirke.

In ihrer vom Bundesminister des Innern vorgetragenen Äußerung bezeichnete die Bundesregierung die Beschwerde als unbegründet und stützte ihre Auffassung auf folgende Argumente: Das EuWG lehne sich an das bewährte und der Bevölkerung vertraute Bundeswahlgesetz mit Fünfprozentklausel an; der Gesichtspunkt der Arbeitsfähigkeit des Parlaments in seiner gruppenmäßigen Ausgestaltung behalte auch dann seine Bedeutung, wenn es noch nicht die Aufgabe habe, eine handlungsfähige und von einer Mehrheit getragene Regierung zu bilden; die Arbeitsfähigkeit des aus nationalen Gruppen von Abgeordneten zusammengesetzten Europäischen Parlaments hänge noch stärker als bei nationalen Parlamenten von der Existenz größerer politischer Gruppen ab; schließlich solle die Sperrklausel auch eine hinreichend wirksame nationale Repräsentanz ermöglichen.

Das Verfassungsgericht bezeichnete die Beschwerden als zulässig, urteilte aber, dass sie nicht begründet seien. Die zentrale Aussage lautete: „Die 5vH-Sperrklausel des $§ 2$ Abs. 6 EuWG ist mit dem Grundgesetz vereinbar, weil sie an dem durch besondere, zwingende Gründe gerechtfertigten Ziel, einer übermäßigen Parteienzersplitterung im Europäischen Parlament entgegenzuwirken, orientiert ist und das Maß des zur Erreichung dieses Ziels Erforderlichen nicht überschreitet."12 Das Gericht bezeichnete den Gleichheitssatz des Grundgesetzes, bezogen sowohl auf die Gleichbewertung aller Staatsbürger bei der Ausübung des Wahlrechts als auch auf die Chancengleichheit der Parteien, als Maßstab für seine Prüfung, ergänzte aber zugleich, dass Differenzierungen - wenn auch nur in einem eng bemessenen Spielraum - möglich seien. Diese bedürften allerdings stets eines besonderen, rechtfertigenden, zwingenden Grundes.

Bei der Entscheidung über den Grad der Differenzierung müsse das Wahlsystem und der Aufgabenbereich des Parlaments berücksichtigt werden. Das durch die Verhältniswahl begünstigte Aufkommen kleiner Parteien könne zu ernsthaften Beeinträchtigungen der Handlungsfähigkeit der zu wählenden Volksvertretung führen. Weil diese Volksvertretung aber nicht nur die in der Wählerschaft vorhandenen politischen Meinungen spiegeln, sondern auch funktionsfähig sein soll, gelte es, das Postulat der Funktionsfähigkeit des Parlaments und die Gebote des grundsätzlich gleichen Erfolgswertes aller Wählerstimmen sowie der gleichen Wettbewerbschancen der politischen Parteien und Wählervereinigungen gegeneinander abzuwägen. Was dabei als zwingender Grund für eine begrenzte Differenzierung anzuerkennen sei, bestimme sich vor allem nach dem Aufgabenbereich der zu wählenden Volksvertretung.

Wenn sich der Gesetzgeber unter Beachtung und nach Abwägung dieser Gesichtspunkte zur Festsetzung einer Sperrklausel entschließe, sei in aller Regel ein Quorum von 5 Prozent verfassungsrechtlich nicht zu beanstanden. Dem Gesetzgeber komme hier ein Ermessensspielraum zu und das Verfassungsgericht dürfe lediglich prüfen, ob die Grenzen des Ermessens überschritten worden seien. Bei Einhaltung dieser Grenzen habe das Gericht die Ausübung des gesetzgeberischen Ermessens zu achten.

Was das Aufgabenspektrum des Europäischen Parlaments betreffe, sei dieses nur sehr bedingt mit dem nationaler Parlamente zu vergleichen. Es handle sich vorrangig um Beratungs- und Kontrollbefugnisse, während elektorale und legislative Funktionen, wie sie sich im parlamentarischen Regierungssystem herausgebildet haben und wahrgenommen werden, nur in ersten Ansätzen vorhanden seien. Das Gericht verwies dazu auf die Mitwirkung des Europäischen Parlaments bei der Bestellung der Mitglieder des Europäischen Rechnungs-

12 BVerfGE 51, 222, Rn. 46. 
hofes; auf das für die Rechtsetzung damals neu eingeführte Konzertierungsverfahren zwischen Europäischem Parlament und Rat (der allerdings das letzte Wort habe) unter Mithilfe der Kommission; auf erste Mitentscheidungsrechte des Europäischen Parlaments über den Haushalt (sie beziehen sich - bei Beachtung von Höchstbeträgen - auf die nicht obligatorischen Ausgaben); auf ein umfangreicher werdendes Instrumentarium von Kontrollrechten, nicht nur gegenüber der Kommission, sondern faktisch auch gegenüber dem Rat; schließlich auf die vom Europäischen Parlament wahrgenommene politische Integrationsfunktion. Ergänzend verwies das Gericht darauf, dass sich Organisation und Arbeitsweise des Europäischen Parlaments nicht wesentlich von derjenigen der nationalen Parlamente der Mitgliedstaaten unterscheiden würden: Die Parlamentsarbeit werde weitgehend in Ausschüssen geleistet und die Abgeordneten hätten sich nicht in nationalen Delegationen, sondern in parteipolitisch ausgerichteten Fraktionen zusammengeschlossen. Der Einfluss fraktionsloser Abgeordneter auf die Willensbildung des Europäischen Parlaments sei indessen gering.

Das Gericht unterstrich, dass das Europäische Parlament mit Blick auf die ihm zukommenden Aufgaben handlungsfähig sein müsse. Dazu gehöre, durch interne Arbeitsteilung allen Mitgliedern die für die Bearbeitung der vielschichtigen Spezialmaterien erforderliche Sachkenntnis zu verschaffen sowie zu überzeugender Mehrheitsbildung in der Lage zu sein. Beides könne durch eine zu starke Aufgliederung des Europäischen Parlaments gefährdet werden. Dies sei ein zwingender Grund für die Rechtfertigung von Vorkehrungen gegen eine übermäßige Parteienzersplitterung. Das Gericht hob sodann die Bedeutung einer engen Verzahnung des Europäischen Parlaments und seiner Mitglieder mit den die Politik der Mitgliedstaaten bestimmenden Parteien heraus. Hierzu könnten im nationalen Bereich unbedeutende Splittergruppen - die als Folge der Sperrklausel bei nationalen Wahlen nicht im nationalen Parlament vertreten seien - wenig oder nichts beitragen. Der Gesichtspunkt eines möglichst homogen zusammengesetzten nationalen Abgeordnetenkontingents falle demgegenüber als Argument nicht entscheidend ins Gewicht.

Das Gericht wiederholte sodann nachdrücklich seine Auffassung, dass dem Gesetzgeber ein Entscheidungs- und Ermessensspielraum zukomme, den das Gericht zu achten habe. Dazu gehöre auch, dass sich der Bundestag an Gegebenheiten und vergleichbaren Regeln in anderen Mitgliedstaaten orientiere. Zwar habe nur Frankreich explizit eine Fünfprozentsperrklausel eingeführt; in einer ganzen Reihe anderer Mitgliedstaaten ergebe sich eine Sperrwirkung aus anderen Gründen: sei es, weil der für die Gewinnung eines Mandats erforderliche Wähleranteil deutlich über der Fünfprozentmarke liege; sei es, dass die für eine Kandidatur neuer Parteien erhobene Kaution oder die Zahl der für die Einreichung einer Kandidatur erforderlichen Unterschriften deutlich über den in Deutschland geltenden Anforderungen liegen würden. Die im deutschen EuWG festgelegte Sperrklausel stehe insofern in Einklang mit vergleichbaren Erschwernissen in den Wahlgesetzen anderer Mitgliedstaaten.

Schließlich griff das Gericht einen weiteren in der Diskussion über die Geltung einer Sperrklausel vorgebrachten Aspekt auf: Von einer Sperrklausel seien nicht nur Splitterparteien betroffen, sondern auch deutsche Fraktionssplitter von großen europäischen Gruppierungen oder Bruderparteien. Nach Auffassung des Gerichts greife auch dieser Einwand im Ergebnis aus zwei Gründen nicht: Zum einen sei das EuWG ein Übergangsgesetz, das nur bis zum Inkrafttreten eines für die Europäischen Gemeinschaften einheitlichen Wahlgesetzes in Kraft bleiben solle; zum zweiten handle es sich bei den hier angesprochenen Gruppen noch um verhältnismäßig lockere Zusammenschlüsse und nicht um organisatorisch verfestigte übernationale Parteien. 
Zusammengefasst zeichnen dieses Urteil die folgenden Hauptmerkmale aus:

- Das Urteil, das die Fünfprozentsperrklausel nach verfassungsmäßiger Prüfung für zulässig erklärt, ergeht einstimmig.

- Dem Prinzip der Gleichheit, Prüfungsmaßstab des Gerichts, wird - verstanden als Wahlrechtsgleichheit der Stimmbürger und als Chancengleichheit der Parteien - ein hoher Rang zugeschrieben. Differenzierungen seien zwar möglich, bedürften aber zu ihrer Rechtfertigung eines besonderen, zwingenden Grundes.

- Diesen Grund sieht das Gericht in der Funktionsfähigkeit des Europäischen Parlaments; die Sperrklausel soll diese gewährleisten und Gefährdungen durch Parteienzersplitterung vermeiden helfen.

- Bei der Entscheidung über die Einführung einer Sperrklausel ist also zwischen dem Postulat der Funktionsfähigkeit des Parlaments und den Grundsätzen der Wahlrechtsgleichheit der Stimmbürger sowie der Chancengleichheit der Parteien abzuwägen; es handelt sich um eine Ermessensentscheidung.

- Das Gericht schreibt dem Gesetzgeber das Recht zu, diese Ermessensentscheidung zu treffen und sieht die Aufgabe des Gerichts nur in der Prüfung der Frage, ob der Gesetzgeber die Grenzen des Ermessens überschritten hat. Das Gericht macht damit deutlich, dass es keinesfalls die Rolle eines Ersatzgesetzgebers wahrnehmen darf.

- Bei der Prüfung der Voraussetzungen für die Funktionsfähigkeit des Europäischen Parlaments geht das Gericht von der vertragsrechtlich definierten Stellung und Funktion des Europäischen Parlaments aus, berücksichtigt aber auch neuere faktische Entwicklungen, die die Tendenz haben, die Position des Europäischen Parlaments zu stärken und es schrittweise - zu einem funktionalen Äquivalent nationaler Parlamente zu machen.

- Die darin deutlich erkennbare bewusste Berücksichtigung des europäischen Kontextes als einem wichtigen Faktor für die Erwägungen des Gerichts und für seine Entscheidung, zeigt sich auch in seinen Ausführungen zu bestimmten Wahlgesetzbestimmungen anderer Mitgliedstaaten, die - als der deutschen Sperrklausel vergleichbare Erschwernisse - ebenfalls dazu beitragen sollen, eine Parteienzersplitterung im Europäischen Parlament zu verhindern.

\section{Die Entscheidung des Bundesverfassungsgerichts vom November 2011 gegen die Fünfprozentsperrklausel im deutschen Europawahlgesetz ${ }^{13}$}

Im Anschluss an die Europawahlen 2009 hatten im Juli und August 2009 drei Stimmbürger Wahlprüfungsbeschwerden, die die Verfassungsmäßigkeit der Fünfprozentsperrklausel betreffen, beim Bundestag eingereicht. Die Beschwerdeführer monierten, weithin übereinstimmend, eine Verletzung der Prinzipien der Wahlrechtsgleichheit und der Chancengleichheit der politischen Parteien. Sie verwiesen auf die Entscheidung des Verfassungsgerichts vom 13. Februar 2008 zum Kommunalwahlrecht in Schleswig-Holstein, ${ }^{14}$ wonach die Zulässigkeit der Fünfprozentsperrklausel nach den jeweils aktuellen Verhältnissen zu beurteilen sei. Dieser Maßstab müsse auch bezüglich der Europawahl 2009 herangezogen werden. Es müsse sich mit hinreichender Wahrscheinlichkeit ergeben, dass die Funktionsfähigkeit ohne die Klausel ernsthaft gefährdet wäre. Die Beschwerdeführer machten weiter geltend, dass

13 Die Ausführungen in diesem Abschnitt folgen dem Text des Beschlusses des Zweiten Senats. Die entsprechenden Textstellen werden - wie im vorherigen Abschnitt - durchgehend in indirekter Rede wiedergegeben und nicht als wörtliche Zitate.

14 BVerfGE 120, $82 \mathrm{ff}$. 
die Klausel nicht geeignet sei, eine Parteienzersplitterung zu verhindern. Ohne Sperrklausel wäre 2009 die Zahl der Parteien nur von 162 auf 169 gestiegen. Und sie unterstrichen, dass das Europäische Parlament seit 1979 trotz einer hohen Anzahl von Parteien funktioniert habe.

Der Bundestag wies die Einsprüche im Juli 2010 mit folgender Begründung zurück: ${ }^{15}$ Der Direktwahlakt der Europäischen Union (in seiner am 25. Juni 2002 geänderten Fassung) sähe die Möglichkeit einer Sperrklausel ausdrücklich vor, ${ }^{16}$ was ein starkes Indiz dafür sei, dass die in Deutschland geltende Sperrklausel nicht verfassungswidrig sei. Weiter führte der Bundestag aus, dass die Begründung des Verfassungsgerichts von 1979 weiterhin zutreffend sei; dass die Rechtfertigung für die Klausel fortbestehe, weil die Gefahr der Zersplitterung noch gewachsen und das Europäische Parlament in seinen Befugnissen in der Zwischenzeit gestärkt worden sei; schließlich: Bei den vom Urteil des Verfassungsgerichts von 2008 betroffenen kommunalen Vertretungen in Schleswig-Holstein handle es sich um Organe der Verwaltung, denen in erster Linie verwaltende Tätigkeiten anvertraut seien, die also einen völlig anderen Charakter als das Europäische Parlament hätten.

Nach dieser Entscheidung des Bundestages wandten sich die Beschwerdeführer mit ihrer Wahlprüfungsbeschwerde an das Bundesverfassungsgericht. Als zusätzliches Argument machten sie geltend, dass der Bundestag in seiner Entscheidung die seit 1979 erfolgten Erfahrungen des Europäischen Parlaments nicht berücksichtigt habe. Der Bundestag verwies in seiner Äußerung dazu darauf, dass die Sicherung der Funktionsfähigkeit einer Volksvertretung als Rechtfertigungsgrund für die mit der Fünfprozentsperrklausel verbundene Wahlgleichheitsbeeinträchtigung anerkannt sei und dass das Verfassungsgericht deshalb den vorrangigen gesetzgeberischen Einschätzungsspielraum zu wahren habe. Das gelte insbesondere auch für das Europäische Parlament, da eine sichere Prognose über die Entwicklungen ohne Sperrklausel nur sehr begrenzt möglich sei. Der vom Gericht 1979 vertretene Rechtfertigungsgrund für die Sperrklausel sei angesichts der erweiterten Kompetenzen des Europäischen Parlaments noch gewichtiger geworden. Ein weiterer Rechtfertigungsgrund sei die politische Rückkoppelung der Abgeordneten an den Mitgliedstaat. In der mündlichen Verhandlung vom 3. Mai 2011 hatten sich auf Bitten des Gerichts, das seinen Erwägungen eine Realanalyse der Arbeits- und Funktionsweise des Europäischen Parlaments zugrunde legen wollte, auch vier vom Präsidenten des Europäischen Parlaments benannte Abgeordnete geäußert.

Das am 9. November 2011 vom Zweiten Senat des Gerichts getroffene Urteil stellte fest, dass die in $\S 2$ Abs. 7 EuWG enthaltene Fünfprozentsperrklausel mit Art. 3 Abs. 1 und Art. 21 Abs. 1 des Grundgesetzes unvereinbar und daher nichtig sei. Der Leitsatz zum Urteil lautete: „Der mit der Fünf-Prozent-Sperrklausel in $\S 2$ Abs. 7 EuWG verbundene schwerwiegende Eingriff in die Grundsätze der Wahlrechtsgleichheit und Chancengleichheit der politischen Parteien ist unter den gegebenen rechtlichen und tatsächlichen Verhältnissen

15 Der Beschluss des Bundestages wurde am 8. Juli 2010 gefasst. Vgl. Zweite Beschlussempfehlung des Wahlprüfungsausschusses zu Einsprüchen gegen die Gültigkeit der Wahl der Abgeordneten des Europäischen Parlaments aus der Bundesrepublik Deutschland am 7. Juni 2009, Bundestagsdrucksache 17/2200, S. 41-47, 55-60 und 107-111; Deutscher Bundestag. Stenografischer Bericht. 55. Sitzung. Berlin, Donnerstag, den 8. Juli 2010, Plenarprotokoll 17/55, S. 5629 C.

16 Art. 3 Direktwahlakt lautet: „Für die Sitzvergabe können die Mitgliedstaaten eine Mindestschwelle festlegen. Diese Schwelle darf jedoch landesweit nicht mehr als 5\% der abgegebenen Stimmen betragen." Vgl. Direktwahlakt. Beschluss und Akt zur Einführung allgemeiner unmittelbarer Wahlen der Abgeordneten des Europäischen Parlaments vom 20. September 1976, zuletzt geändert durch Beschluss des Rates vom 25. Juni 2002 und 23. September 2002, in: Bundesgesetzblatt Teil II, Nr. 21 vom 20. August 2003, S. 810-814 und Bundesgesetzblatt Teil II, Nr. 12 vom 27. April 2004, S. 520. 
nicht zu rechtfertigen. " ${ }^{17}$ Die Entscheidung war mit 5 gegen 3 Stimmen ergangen, wobei das Ergebnis von einem Mitglied des Senats aus abweichenden Gründen - die, wie kritisch kommentiert wurde, nicht öffentlich erläutert wurden, also unbekannt bleiben - mitgetragen wurde. Zwei Richter (Udo Di Fabio und Rudolf Mellinghoff) haben ihre Abweichende Meinung zum Urteil schriftlich formuliert. Sie ist Teil des Urteils.

Die Begründung des Urteils, die - wie erwähnt - nur von vier Mitgliedern des Zweiten Senats getragen wird, bezieht sich auf die zwei im Leitsatz genannten Punkte: die rechtlichen und tatsächlichen Verhältnisse. Sie sind Prüfungsmaßstäbe für das Gericht.

\section{Die rechtlichen Verhältnisse als Prüfungsmaßstab}

Was den ersten Punkt - die rechtlichen Verhältnisse - betrifft, argumentiert die Begründung wie folgt:

Das EuWG sei, als deutsches Bundesrecht, am Grundgesetz mit den beiden darin enthaltenen Grundsätzen der Wahlrechtsgleichheit und Chancengleichheit der politischen Parteien zu messen. Der Direktwahlakt eröffne den Mitgliedstaaten lediglich die Möglichkeit, eine Mindestschwelle für die Sitzvergabe von landesweit bis zu 5 Prozent der abgegebenen Stimmen festzulegen, stelle aber keine verpflichtende europarechtliche Vorgabe dar und lasse daher die Reichweite der innerstaatlichen Überprüfung der Vereinbarkeit einer solchen Regelung mit den durch das Grundgesetz verbürgten Wahlgrundsätzen unberührt.

Was den Grundsatz der Wahlgleichheit angehe, sei der deutsche Gesetzgeber mit Blick auf die europarechtlich vorgegebene Verhältniswahl verpflichtet, bei der Ausgestaltung des EuWG sowohl die Zähl- als auch die Erfolgswertgleichheit der Wählerstimmen sicherzustellen, weil bei diesem Wahlsystem jeder Wähler den gleichen Einfluss auf die Zusammensetzung der zu wählenden Vertretung haben müsse. Bei der Europawahl 2009 in Deutschland hätten aber rund 2,8 Millionen - also etwa 10 Prozent - der gültig abgegebenen Stimmen keinen Erfolgswert gehabt.

Was den Grundsatz der Chancengleichheit der Parteien angehe, sei Gleichheit in einem strikten und formalen Sinne zu fordern. Das bedeute, dass jeder Partei die gleichen Möglichkeiten im gesamten Wahlverfahren und damit gleiche Chancen bei der Verteilung der Sitze eingeräumt werden müssten. Bei der Europawahl 2009 in Deutschland seien aber sieben Parteien und sonstige politische Vereinigungen unberücksichtigt geblieben, die ohne die Fünfprozentklausel bei der Sitzverteilung zum Zuge gekommen wären. Diese Berechnungen würden im Übrigen etwaige Auswirkungen der Sperrklausel auf das Wählerverhalten unberücksichtigt lassen.

Beide Grundsätze würden zwar keinem absoluten Differenzierungsverbot unterliegen; dem Gesetzgeber würde aber nur ein eng begrenzter Spielraum für Differenzierungen verbleiben, wobei ein strenger Maßstab anzulegen sei. Dieser sei auf die mit der Wahl verfolgten Ziele zu beziehen, zu denen die Sicherung des Charakters der Wahl als eines Integrationsvorgangs bei der Willensbildung des Volkes und, damit zusammenhängend, die Sicherung der Funktionsfähigkeit der zu wählenden Volksvertretung gehören würden.

Differenzierende Regelungen müssten zur Verfolgung ihrer Zwecke geeignet und erforderlich sein. Der Gesetzgeber müsse sich bei seiner Einschätzung und Bewertung nicht an abstrakt konstruierten Fallgestaltungen, sondern an der politischen Wirklichkeit orientieren, also den aktuellen Verhältnissen. Eine einmal als zulässig angesehene Sperrklausel könne

17 BVerfGE 129, 130, Leitsatz. 
und dürfe deshalb nicht als für alle Zeiten verfassungsrechtlich unbedenklich eingeschätzt werden. Maßgeblich für die Frage der weiteren Beibehaltung der Sperrklausel seien allein die aktuellen Verhältnisse.

Obwohl das Gericht nicht die Aufgabe des Gesetzgebers zu übernehmen oder eigene Zweckmäßigkeitserwägungen an die Stelle derjenigen des Gesetzgebers zu setzen habe, sei die Ausgestaltung des Wahlrechts aus einem weiteren Grund einer strikten Kontrolle zu unterziehen: Mit Regelungen, die die Bedingungen der politischen Konkurrenz berühren, würde eine parlamentarische Mehrheit gewissermaßen in eigener Sache - geleitet vom Ziel des eigenen Machterhalts - tätig werden.

Schließlich habe das Gericht in seiner Entscheidung vom 13. Februar 2008 zur Fünfprozentsperrklausel im Kommunalwahlrecht Schleswig-Holsteins, wo Verhältniswahl gilt, die Prüfungsmaßstäbe konkretisiert. Danach beruhe der Einsatz einer Sperrklausel auf der Einschätzung des Gesetzgebers von der Wahrscheinlichkeit des Einzugs von Splitterparteien, dadurch zu erwartender Funktionsstörungen und deren Gewicht für die Aufgabenerfüllung der Volksvertretung. Bei dieser Prognoseentscheidung dürfe der Gesetzgeber nicht auf die Feststellung der rein theoretischen Möglichkeit einer Beeinträchtigung der Funktionsfähigkeit der Volksvertretung zur Rechtfertigung des Eingriffs abstellen; nur die mit einiger Wahrscheinlichkeit zu erwartende Beeinträchtigung der Funktionsfähigkeit der Vertretungsorgane, was nur mit Blick auf die tatsächlichen Verhältnisse möglich sei, könne die Fünfprozentklausel rechtfertigen.

\section{Die tatsächlichen Verhältnisse als Prüfungsmaßstab}

Was diese tatsächlichen Verhältnisse - den zweiten Prüfungsmaßstab - angeht, widerspricht die Begründung der Einschätzung des Gesetzgebers, dass das Europäische Parlament mit dem Wegfall der Fünfprozentsperrklausel in seiner Funktionsfähigkeit beeinträchtigt würde. Eine solche Einschätzung könne sich nicht auf ausreichende tatsächliche Grundlagen stützen und trage den spezifischen Arbeitsbedingungen des Europäischen Parlaments und seiner Aufgabenstellung nicht angemessen Rechnung. Im Einzelnen argumentiert die Urteilsbegründung wie folgt:

Bereits jetzt sei das Europäische Parlament stark fragmentiert; unter den mehr als 160 Parteien seien viele, die nur über einen oder zwei Sitze verfügen. Präzise Prognosen über die künftige Zusammensetzung des Europäischen Parlaments seien nicht möglich, weil der Erfolg von Parteien von einer Reihe von Faktoren, die von Mitgliedstaat zu Mitgliedstaat recht unterschiedlich seien, bestimmt würde.

Es sei nicht erkennbar, dass durch die Zunahme von Parteien mit einem oder zwei Abgeordneten im Europäischen Parlament dessen Funktionsfähigkeit mit der erforderlichen Wahrscheinlichkeit beeinträchtigt würde. Die im Europäischen Parlament gebildeten Fraktionen würden, wie die Erfahrung zeige, über eine erhebliche Integrationskraft verfügen, die durch den Einzug weiterer Parteien ebenso wenig grundsätzlich infrage gestellt würde wie ihre Absprachefähigkeit. Was diese betrifft, verweist die Urteilsbegründung auf die bisherige parlamentarische Praxis, die durch die Zusammenarbeit der beiden großen Fraktionen, aber auch durch die Bildung anderer und neuer Mehrheiten geprägt sei. Das Risiko einer Erschwerung der Mehrheitsbildung - wie sie die vier deutschen Mitglieder des Europäischen Parlaments erwarten, die sich in der mündlichen Verhandlung des Gerichts dazu geäußert 
hatten ${ }^{18}$ - sei, auch wenn es um die Bildung qualifizierter Mehrheiten gehe, mit der Gefahr einer Funktionsbeeinträchtigung nicht gleichzusetzen.

Während die Fünfprozentsperrklausel bei der Wahl zum Deutschen Bundestag ihre Rechtfertigung im Wesentlichen darin finde, dass die Bildung einer stabilen Mehrheit für die Wahl einer handlungsfähigen Regierung und deren fortlaufende Unterstützung nötig sei und dieses Ziel durch eine Zersplitterung des Parlaments in viele kleine Gruppen gefährdet würde, bestehe eine vergleichbare Interessenlage auf europäischer Ebene nach den europäischen Verträgen nicht:

(1) Zwar werde der Kommissionspräsident vom Europäischen Parlament mit der Mehrheit seiner Mitglieder gewählt und müsse sich das Gesamtkollegium der Kommission einem Zustimmungsvotum des Europäischen Parlaments stellen; bei der Erfüllung ihrer Aufgaben seien aber die Kommission und ihr Präsident nicht auf seine weitere Zustimmung angewiesen.

(2) Die Rechtsetzung im ordentlichen Gesetzgebungsverfahren erfordere keine mehrheitsgetragene Zustimmung des Parlaments; für das Zustandekommen eines Rechtsaktes sei die Zustimmung des Europäischen Parlaments mithin nicht zwingend. Der Rechtsakt gelte nämlich auch dann als erlassen, wenn sich das Europäische Parlament in der zweiten Lesung zum Standpunkt des Rates nicht äußere oder den Ratsvorschlag nicht mit der Mehrheit seiner Mitglieder ablehne. Weil also die unionale Gesetzgebung nach dem Primärrecht so konzipiert sei, dass sie nicht von bestimmten Mehrheitsverhältnissen im Europäischen Parlament abhänge, entfalle ein zentraler Grund für die Rechtfertigung der Fünfprozentsperrklausel.

(3) Ähnlich verhalte es sich bei der Erstellung des Jahreshaushaltsplans. Das Europäische Parlament könne zwar Änderungen am Standpunkt des Rates mit der Mehrheit seiner Mitglieder beschließen und es könne - im Rahmen des Vermittlungsverfahrens - den von ihm gebilligten gemeinsamen Entwurf auch gegen den Willen des Rates als Haushaltsgesetz durchsetzen, wofür es einer besonders qualifizierten Mehrheit (drei Fünftel der abgegebenen Stimmen, die zugleich die Mehrheit der Mitglieder des Europäischen Parlaments darstellen) bedürfe. Fasse das Europäische Parlament zum Standpunkt des Rates hingegen keinen Beschluss, so gelte der Haushaltsplan als erlassen; insoweit könne auch der Haushaltsplan ohne die Zustimmung des Europäischen Parlaments zustande kommen.

(4) Schließlich folge aus dem Umstand, dass bestimmte, in den Verträgen ausdrücklich aufgeführte Rechtsetzungsakte nur mit Zustimmung des Europäischen Parlaments nach besonderen Gesetzgebungsverfahren erlassen werden könnten, nicht die Notwendigkeit einer stabilen Parlamentsmehrheit. Eine generelle Blockade parlamentarischer Tätigkeit drohe nicht, wenn in einzelnen Fällen - hier wird auf die von den Verträgen vorgesehenen gänzlich unterschiedlichen Fallgestaltungen verwiesen - eine Mehrheit für die Zustimmung nicht zustande käme. Hiervon sei auch im Hinblick auf die Zustimmungsvorbehalte bei sogenannten konstitutionellen Rechtsakten auszugehen.

(5) Von vornherein ausgeschlossen werden könne, dass mit der zugrunde zu legenden Zunahme kleiner Parteien das Europäische Parlament in der Wahrnehmung seiner Aufgaben der politischen Kontrolle behindert werde. So entscheide das Europäische Parlament bei der Kontrolle des Haushaltsvollzugs der Kommission mit der Mehrheit der abgegebenen Stimmen über die Entlastung, wobei deren Verweigerung als Misstrauensvotum gegenüber der

18 Elmar Brok (CDU), Gerald Häfner (Bündnis 90/Die Grünen), Klaus-Heiner Lehne (CDU) und Bernhard Rapkay (SPD). 
Kommission gelte; weil dieses rechtlich allerdings als folgenlos angesehen werde, bedürfe es auch insoweit keiner mehrheitsstabilisierenden Maßnahmen.

Schließlich nimmt die Begründung zu Gesichtspunkten Stellung, die unabhängig von der Behauptung einer rechtserheblichen Funktionsbeeinträchtigung des Europäischen Parlaments zugunsten der Aufrechterhaltung der Fünfprozentsperrklausel vorgetragen worden waren; die Begründung führt dazu aus, dass auch diese Gesichtspunkte nicht geeignet seien, den Eingriff in die Wahl- und Chancengleichheit zu rechtfertigen.

Dabei geht es, zum ersten, um die These, die Sperrklausel sei zur Gewährleistung einer wirksamen Vertretung der deutschen Interessen im Europäischen Parlament erforderlich. Es liege, so heißt es in der Begründung, auf der Hand, dass damit kein tragfähiger Grund für eine wahlrechtliche Ungleichbehandlung benannt sei. Der Gesetzgeber dürfe größere Parteien nicht allein deshalb bevorzugen, weil sie ihre Auffassungen auf europäischer Ebene voraussichtlich mit größerer Aussicht auf Erfolg als kleine Parteien einbringen könnten.

Entsprechendes gelte, zum zweiten, für den vom Deutschen Bundestag angeführten Gesichtspunkt der politischen Rückkoppelung der Abgeordneten des deutschen Kontingents im Europäischen Parlament an den Deutschen Bundestag, soweit damit die Wirksamkeit der Vertretung deutscher Interessen sichergestellt werden soll. Zwar handle es sich bei diesem Gesichtspunkt um ein - als solches durchaus gewichtiges - politisches Anliegen; verfassungsrechtliche Bedeutung komme ihm jedoch nicht zu.

\section{Die Kritik an der Entscheidung des Gerichts vom November 2011 gegen die Fünfprozentsperrklausel und an der Begründung für diese Entscheidung}

Im Sondervotum von zwei Mitgliedern des Zweiten Senats, der Richter Di Fabio und Mellinghoff, heißt es einleitend: „Wir tragen die Entscheidung in Ergebnis und Begründung nicht mit. Der Senat gewichtet durch eine zu formelhafte Anlegung der Prüfungsmaßstäbe den Eingriff in die Wahlrechtsgleichheit und Chancengleichheit politischer Parteien nicht überzeugend, zieht den Gestaltungsspielraum des Wahlgesetzgebers zu eng und nimmt eine mögliche Funktionsbeeinträchtigung des Europaparlaments trotz dessen gewachsener politischer Verantwortung in Kauf. [...] Hat das Bundesverfassungsgericht noch im Jahr 1979 die Fünf-Prozent-Sperrklausel für die Europawahl als gerechtfertigt angesehen [...], so hält der Senat heute trotz abnehmender praktischer Wirkung der Sperrklausel [...] und trotz beträchtlicher Kompetenzzuwächse sowie einer deutlich gestiegenen politischen Bedeutung des Europaparlaments [...] die Sperrklausel für nicht mehr gerechtfertigt, ohne dass hinreichend offengelegt wird, inwieweit sich die Maßstäbe der Beurteilung verändert haben." ${ }^{19}$

Diese Abweichende Meinung, ,die sich in Duktus und Inhalt entschieden von der Mehrheitsmeinung absetzt" ${ }^{20}$ ist eine deutliche Kritik an der Entscheidung und ihrer Begründung. Zur Begründung ihrer Kritik, die im Folgenden referiert wird, ${ }^{21}$ führen die zwei Richter eine Reihe von Argumenten ins Feld. Diese beziehen sich erstens auf die Frage nach der Eingriffsintensität der Fünfprozentsperrklausel, zweitens auf die Funktionsfähigkeit des Europäischen Parlaments und ganz generell auf die Frage nach der Rolle des Gerichts im Verhältnis zum Gesetzgeber und dessen Gestaltungsspielraum.

19 BVerfGE 129, 130, Rn. 147-148.

20 Bernd Grzeszick: Demokratie und Wahlen im europäischen Verbund der Parlamente - Zum Urteil des BVerfG über Sperrklauseln bei Wahlen zum Europäischen Parlament, in: Europarecht 6/2012, S. 667-681.

21 Die nachfolgenden Ausführungen folgen dem Text der Abweichenden Meinung. Die entsprechenden Textstellen werden - wie in den beiden vorherigen Abschnitten - in indirekter Rede wiedergegeben und, bis auf wenige Ausnahmen, nicht als wörtliche Zitate. 
Zum ersten Fragenkomplex wird ausgeführt, dass die Sperrklausel eine ergänzende Regelung zum Verhältniswahlrecht sei, in diesem Kontext als Erfolgswertdifferenzierung zu sehen sei und in ihrer Eingriffsintensität entsprechend gewichtet werden müsse. Verglichen mit einem Mehrheitswahlsystem sei das Verhältniswahlsystem mit der Annexbedingung einer Fünfprozentsperrklausel aus Sicht der Erfolgswertgleichheit weniger einschneidend. Beide Wahlsysteme seien vom Grundgesetz erlaubt und die insofern im Hinblick auf das Wahlsystem offene Verfassung verleihe dem Bundesverfassungsgericht deshalb kein Mandat, „bei der konzeptionellen Ausgestaltung eines Wahlsystems allzu feingliedrig mit dem Gleichheitssatz zu prüfen, fallweise zu korrigieren und so allmählich in die Rolle des Wahlgesetzgebers zu schlüpfen." ${ }^{22}$ Der Wahlgesetzgeber sei nicht zur Ausgestaltung eines reinen Modells - hier also der Verhältniswahl ohne Modifikation mittels Sperrklausel - genötigt, „so dass die Fünf-Prozent-Klausel als grundsätzlich zulässiges Regulativ gegen die Nachteile einer hohen Abbildungsleistung der verschiedenen auch kleineren und kleinsten Parteien gewichtet werden" 23 müsse.

Was die Eingriffsintensität der Fünfprozentklausel bei der Europawahl angehe, so ergebe sich keine Besonderheit im Vergleich zu anderen Wahlen, die eine besonders strenge Prüfung gebieten würde. Ganz im Gegenteil verringere sich die Eingriffsintensität - etwa wegen der Zunahme der Volatilität der Wählerschaft - und zudem sei die Fünfprozenthürde heute nicht mehr das Hindernis wie vor drei Jahrzehnten, was man am Wahlerfolg ganz neu gegründeter Parteien sehen könne, die gleichsam aus dem Stand heraus Sperrklauseln überwinden würden. Eine strengere Prüfung sei schließlich auch nicht deshalb erforderlich, weil Parteien mit der Bestimmung einer Sperrklausel gleichsam in eigener Sache - auf Kosten konkurrierender (kleinerer) Parteien - entscheiden würden; dies könne hier nicht unterstellt werden.

Was die Funktionsfähigkeit des Europäischen Parlaments, den zweiten Fragenkomplex, angeht, sei die Fünfprozentklausel auch sachlich gerechtfertigt, um nämlich auch für das deutsche Kontingent eine zu weitgehende Zersplitterung der im Europäischen Parlament vertretenen Parteien zu verhindern. Deutschland trage dafür, wie auch andere Mitgliedstaaten, nur eine Teilverantwortung; dabei komme dem Beitrag der Mitgliedstaaten mit größeren Mandatskontingenten eine besondere Rolle zu, ohne dass es sich dabei um förmliche Sperrklauseln handeln müsse. So würden die Wahlsysteme einer Reihe von Mitgliedstaaten wahltechnische Ausgestaltungen enthalten, die im Hinblick auf ihre Konzentrationswirkung den Sperrklauseln vergleichbar seien. Vor diesem Hintergrund werde mit der isolierten Aufhebung der deutschen Fünfprozentklausel im europäischen Umfeld ein Sonderweg beschritten.

Die Wahrung der Funktionsfähigkeit sei gerade im Fall des Europäischen Parlaments keine Frage des Entweder-Oder, sondern eines Mehr oder Weniger an Handlungsfähigkeit, wobei die funktionalen Besonderheiten des Europäischen Parlaments - also eben nicht die Fähigkeit zur Bildung einer Regierung durch die Parlamentsmehrheit - angemessen gewürdigt werden müssten. Bereits die Verringerung möglicher Funktionsbeeinträchtigungen sei ein Grund für die Rechtfertigung der Sperrklausel. Der Senat verenge aber den Begriff der Funktionsbeeinträchtigung letztlich auf Funktionsunfähigkeit. Das Europäische Parlament benötige aber Handlungsfähigkeit auch und gerade dort, wo es in einem verhandelnden Mehrebenensystem etwa im Verfahren der Mitentscheidung eine Position durchsetzen wolle.

In der mündlichen Verhandlung hätten Abgeordnete des Europäischen Parlaments die schon früher in der Wissenschaft geäußerte Ansicht, dass große Fraktionen mit der Fähigkeit

22 BVerfGE 129, 130, Rn. 154.

23 Ebenda. 
zum übergreifenden Konsens notwendig seien, um politische Stärke zu entwickeln, nachdrücklich bestätigt. Eine durch Verzicht auf die Sperrklausel sicher zu erwartende weitere Zergliederung und zahlenmäßige Schwächung gerade auch der großen Fraktionen werde die ohnehin bestehenden Organisationsprobleme bei der Willensbildung verstärken. Gegen diese Einschätzung, die auch der Wahlgesetzgeber seiner Entscheidung für die Sperrklausel zugrunde gelegt habe, könne der Senat keine eigene Beurteilung setzen, ohne den Prüfungsmaßstab verschärfen zu müssen oder die Tätigkeit der Mitglieder des Europäischen Parlaments ohne praktische Anschauung zu vermessen.

Im Zusammenspiel mit Kommission und Rat komme es für das Europäische Parlament darauf an, mehrheitsfähige Willensbildung in den eigenen Reihen herbeizuführen. Auch wenn das bisher, trotz großer Heterogenität, gelungen sei, könne die Devise nicht lauten, man könne dem Europäischen Parlament diesbezüglich noch mehr zumuten. Ein weiterer Gesichtspunkt komme hinzu: Das Europäische Parlament sei in Sachthemen politisch kontroverser geworden. Das setze aber voraus, dass die größeren Fraktionen den Standpunkt ihrer Abgeordneten erfolgreich bündeln und gegenüber anderen Fraktionen vertreten könnten. Und das Europäische Parlament befinde sich nach Inkrafttreten des Vertrags von Lissabon in einer neuen Phase seiner Entwicklung, was die Vertreter des Europäischen Parlaments in der mündlichen Verhandlung gleichfalls unterstrichen hätten.

Diese in der Abweichenden Meinung vorgetragene Kritik an der Entscheidung des Zweiten Senats vom November 2011 gegen die Beibehaltung der Fünfprozentsperrklausel kann mit weiteren Argumenten und Gesichtspunkten ergänzt und verstärkt werden:

So moniert Schönberger, ${ }^{24}$ dass die Entscheidung des Zweiten Senats der „Gemengelage von Unionsrecht und mitgliedstaatlichem Recht bei der Ausgestaltung der Wahl zum Europäischen Parlament ${ }^{“ 25}$ nicht Rechnung getragen habe. Der Direktwahlakt, der einen „Unbedenklichkeitskorridor“26 für eine Sperrklausel bis zur maximalen Höhe von 5 Prozent geschaffen habe, würde ausgeblendet. Die Überprüfung der Sperrklausel am Maßstab des Grundgesetzes verlange aber die sorgfältige Einordnung in das normative Gesamtumfeld, also die Berücksichtigung der Gemengelage; daran gehe der Zweite Senat „mit großer Nonchalance" 27 vorbei.

Weiterhin kritisiert Schönberger die „Einebnung der grundgesetzlichen Maßstäbe“28: Der Zweite Senat verzichte darauf, einen eigenständigen verfassungsrechtlichen Maßstab für die Prüfung des deutschen Direktwahlgesetzes zu bilden. Stattdessen gehe der Zweite Senat den umgekehrten Weg: Er „,behauptet einen von konkreten Verfassungsnormen weitgehend abgelösten universellen Standard der Wahlrechtsgleichheit für alle Wahlen in Deutschland und will die Eigenheiten der jeweiligen Volksvertretung lediglich bei der Rechtfertigung von Differenzierungen im Hinblick auf deren Funktionen berücksichtigt sehen. "29 Insbesondere für die Europawahl sei aber das gegenteilige Vorgehen nötig: „Es bedarf einer differenzierten Herausbildung grundgesetzlicher Maßstäbe, die von vornherein dem Charakter des Europäischen Parlaments als Unionsorgan, den unionsrechtlichen Vorprägungen und der dienenden Funktion des mitgliedstaatlichen Wahlrechts Rechnung tragen. " ${ }^{\text {" }}$ Was diesen letzten

24 Schönberger: Das Bundesverfassungsgericht und die Fünf-Prozent-Klausel, 2012, S. 80-86.

25 Ebenda, S. 81.

26 Ebenda, S. 82.

27 Ebenda, S. 81.

28 Ebenda, S. 82.

29 Ebenda.

30 Ebenda, S. 83. 
Aspekt betrifft, bescheinigt Schönberger dem Urteil „fehlende wahlrechtliche Institutionensorge. " ${ }^{31}$

Kritikwürdig sei, so Schönberger, auch die fehlende Auseinandersetzung mit der Entscheidung von 1979, die die Sperrklausel für verfassungsgemäß erklärt hatte. Es stehe dem Gericht frei, gut dreißig Jahre später zu einer aus seiner Sicht besser begründeten gegenteiligen Rechtsauffassung zu gelangen. „Aber gerade dann wäre eine eingehende Auseinandersetzung mit der sehr differenzierten Argumentation der Vorgängerentscheidung am Platz gewesen. ${ }^{\text {32 } 2}$ Das Gericht bleibe aber die Antwort auf die Frage schuldig, welches denn die geänderten Verhältnisse sein sollen, die heute eine Rechtfertigung der Sperrklausel nicht länger erlauben würden. Und Schönberger fügt, in Übereinstimmung mit anderen Kritikern der Entscheidung (zu denen auch der Verfasser dieses Beitrags gehört), hinzu: „Tatsächlich ist es vielmehr so, dass alle diejenigen Umstände, die sich seit der damaligen Entscheidung gewandelt haben, heute eher stärker für die Sperrklausel sprechen als dies damals der Fall war."“33

Jeder, der die Entwicklung des EU-Entscheidungssystems - insbesondere auch hinsichtlich der Rolle des Europäischen Parlaments - in den letzten Jahren verfolgt hat, wird registriert haben, welche tiefgreifende Veränderungen erfolgt sind. Das gilt insbesondere mit Blick auf das mit dem Vertrag von Lissabon eingeführte neue Regelwerk. Nicht nur ist das Europäische Parlament in seiner rechtlichen und politischen Stellung signifikant gestärkt worden, ${ }^{34}$ auch den nationalen Parlamenten ist - als bemerkenswerte, aber wohl noch nicht ausreichend beachtete Neuerung - die Rolle als institutioneller Mitspieler zugeschrieben worden. ${ }^{35}$ Dieser letztere Aspekt verdient, wie zu zeigen sein wird, gerade in dem hier erörterten Kontext besondere Aufmerksamkeit. Neuerungen, die vorrangig für die Fraktionen des Europäischen Parlaments relevant sind, wurden auch in dessen politischem Umfeld eingeführt: Dabei handelt es sich um das durch Verordnung von Rat und Europäischem Parlament im November 2003 beschlossene Parteienstatut, ${ }^{36}$ welches „Regelungen für die Parteien auf europäischer Ebene und ihre Finanzierung“ eingeführt hat. Die im Dezember 2007 beschlossene Verordnung zur Änderung des Parteienstatuts sieht die Möglichkeit zur finanziellen Unterstützung von Stiftungen auf EU-Ebene vor, die den Parteien auf europäischer Ebene angeschlossen sind. ${ }^{37}$ Die Aktivitäten dieser Parteien auf europäischer Ebene ${ }^{38}$ beziehen sich mit ihren transnationalen Kommunikationsbeziehungen auch auf die jeweiligen Fraktionen im Europäischen Parlament, die im Übrigen auch in der Organisationsstruktur der europäischen Parteien repräsentiert sind. Sie tragen dazu bei, das politische Profil der

31 Ebenda, S. 86.

32 Ebenda, S. 85.

33 Ebenda.

34 Stichworte sind hier vor allem: das Verfahren der Mitentscheidung bei der Rechtsetzung; die gleichberechtigte Rolle des Europäischen Parlaments im Haushaltsverfahren; die Rechte des Europäischen Parlaments bei der Bestellung des Präsidenten der Kommission und des Kollegiums der Kommission.

35 Vgl. dazu beispielsweise Rudolf Hrbek: The Role of National Parliaments in the EU, in: Hermann-Josef Blanke/ Stelio Mangiameli (Hrsg.): The European Union after Lisbon. Constitutional Basis, Economic Order and External Action, Berlin/Heidelberg 2012, S. 129-157.

36 Verordnung (EG) Nr. 2004/2003 des Europäischen Parlaments und des Rates vom 4. November 2003 über die Regelungen für die politischen Parteien auf europäischer Ebene und ihre Finanzierung, in: Amtsblatt der EU, Nr. L 297 vom 15. November 2003, S. 1-4.

37 Verordnung (EG) Nr. 1524/2007 des Europäischen Parlaments und des Rates vom 18. Dezember 2007 zur Änderung der Verordnung (EG) Nr. 2004/2003 über die Regelungen für die politischen Parteien auf europäischer Ebene und ihre Finanzierung, in: Amtsblatt der EU, Nr. L 343 vom 27. Dezember 2007, S. 5-8.

38 Vgl. dazu Jürgen Mittag/Janosch Steuwer (Hrsg.): Politische Parteien in der EU, Wien 2010. 
Fraktionen zu schärfen und zur Politisierung der im Wesentlichen von den Fraktionen getragenen und bestimmten Arbeit des Europäischen Parlaments beizutragen.

Die vom Zweiten Senat als für die von ihm vorzunehmende Prüfung der Zulässigkeit der Fünfprozentsperrklausel als wesentlich und letztlich ausschlaggebend bezeichnete Orientierung an der politischen Wirklichkeit ${ }^{39}$ war, bezogen auf die eben genannten neuen Gegebenheiten, recht unvollständig und selektiv. ${ }^{40}$ Er hat die von den deutschen Europaabgeordneten in der öffentlichen Anhörung mit Nachdruck vorgetragenen übereinstimmenden Aussagen und Einschätzungen, die übrigens auch dem Votum des Bundestages zugrunde lagen, ganz offensichtlich als für seine eigene Urteilsbildung irrelevant angesehen. Wo der Zweite Senat solche Neuerungen genannt hat, waren die von ihm gezogenen Schlussfolgerungen erstaunlich und ganz und gar nicht überzeugend.

Der Zweite Senat basiert die Begründungen für seine Entscheidung an manchen Stellen auf bloße Annahmen und Mutmaßungen. Das gilt etwa für Aussagen zur künftigen Integrationskraft der Fraktionen. Diese werde im Licht der bisherigen Erfahrungen ausreichend stark sein, um gravierende Funktionsbeeinträchtigungen zu verhindern. ${ }^{41}$ Das gilt auch für Aussagen zu künftigen Absprachen und Bündnissen zur Mehrheitsgewinnung. Zwar sei die parlamentarische Praxis durch eine Zusammenarbeit der beiden größten Fraktionen geprägt, eine höhere Anzahl von Abgeordneten kleiner Parteien stehe indessen einer geordneten Mehrheitsbildung nicht im Weg, denn bei den ganz unterschiedlichen Abstimmungsgegenständen würden sich ,immer wieder neue Mehrheiten bilden. “42

Nicht überzeugend fällt auch die Argumentation des Zweiten Senats zum Verfahren der Mitentscheidung aus. Die Rechtsetzung in diesem Verfahren erfordere keine mehrheitsgetragene Zustimmung des Parlaments. ${ }^{43}$ Ein Rechtsakt, den der Rat festlege und dem Europäischen Parlament übermittle, gelte auch dann als erlassen, „wenn sich das Parlament in der zweiten Lesung zum Standpunkt des Rates nicht äußert oder den Ratsvorschlag nicht mit der Mehrheit seiner Mitglieder ablehnt. “44 Hier muss sich der Zweite Senat die Frage nach seiner Einschätzung des Selbstverständnisses des Europäischen Parlaments und seiner Mitglieder gefallen lassen. Das Bild von einem Parlament - hier dem Europäischen Parlament -, das nicht darauf bedacht ist, Entscheidungen zu beeinflussen und mitzubestimmen, hat mit der Wirklichkeit nichts zu tun und ist abwegig. Gleiches gilt für die Aussagen zur Erstellung des Jahreshaushaltsplans, wenn es heißt: „Fasst das Parlament zum Standpunkt des Rates [...] hingegen keinen Beschluss, so gilt der Haushaltsplan als erlassen. “45

Ohne jede Wirklichkeitsorientierung ist schließlich die Position des Zweiten Senats zum Gesichtspunkt der politischen Rückkoppelung der deutschen Mitglieder des Europäischen Parlaments an den Deutschen Bundestag. ${ }^{46}$ Diesem - als solchem durchaus gewichtigen politischen Anliegen komme keine verfassungsrechtliche Bedeutung zu. Diese Aussage von einem Argument kann nicht die Rede sein - ignoriert die im Primärrecht enthaltenen

39 BVerfGE 129, 130, Rn. 89.

40 Martin Morlok, der der Entscheidung des Zweiten Senats ,im Ergebnis und weitestgehend auch in der Begründung“ zustimmt, bezeichnet hingegen ,die - offen deklarierte - Wirklichkeitsorientierung“ des Urteils als „,bemerkenswert“. Vgl. Martin Morlok: Chancengleichheit ernstgenommen - Die Entscheidung des Bundesverfassungsgerichts zur Fünf-Prozent-Klausel bei der Europawahl, in: JuristenZeitung 2/2012, S. 76-80, hier S. 78.

41 BVerfGE 129, 130, Rn. 105 und 106.

42 Ebenda, Rn. 110 sowie insgesamt zu diesem Punkt die Rn. 106-109.

43 Ebenda, Rn. 120.

44 Ebenda, Rn. 122.

45 Ebenda, Rn. 123.

46 Ebenda, Rn. 129. 
Bestimmungen über die Rolle nationaler Parlamente bei Entscheidungsprozessen in der Europäischen Union, zu der ausdrücklich auch Bestimmungen zur Zusammenarbeit zwischen dem Europäischen Parlament und den nationalen Parlamenten gehören. ${ }^{47}$ Und sie ignoriert die Realität der seit Jahren praktizierten und sich stetig verdichtenden ganz praktischen Kommunikations- und Kooperationsbeziehungen zwischen dem Deutschen Bundestag (also seinen Fraktionen und Ausschüssen) und dem Europäischen Parlament, vorrangig seinen deutschen Mitgliedern. ${ }^{48}$ Man wird in diesem Zusammenhang auch daran erinnern dürfen, dass dem Deutschen Bundestag ausdrücklich eine „Integrationsverantwortung“49 zugeschrieben wird und zukommt, zu der sich das Bundesverfassungsgericht in seiner LissabonEntscheidung ebenfalls geäußert hat. ${ }^{50}$

Im Urteil des Zweiten Senats zeigt sich ein erhebliches Maß an Unverständnis für die Besonderheiten des immer komplexer gewordenen Verhandlungssystems in der Europäischen Union, insbesondere auch für die intra- und interinstitutionellen Kommunikationsbeziehungen, an denen das Europäische Parlament mit seinen Fraktionen, Ausschüssen und einzelnen Mitgliedern immer stärker und wirkungsvoller beteiligt ist. Dem trägt die Entscheidung des Zweiten Senats, die Fünfprozentsperrklausel als nicht verfassungsgemäß zu erklären, nicht Rechnung.

\section{Das mit Blick auf die nächsten Europawahlen 2014 im Juni 2013 vom Deutschen Bundestag geänderte Europawahlgesetz}

Nachdem aufgrund der Entscheidung des Zweiten Senats des Bundesverfassungsgerichts vom November 2011 die Fünfprozentsperrklausel nicht mehr gültig war, musste das deutsche EuWG novelliert werden. Eine weitere Anpassung war als Folge einer unionsrechtlichen Vorgabe im Vertrag über die Europäische Union (EUV) ${ }^{51}$ erforderlich, wonach künftig kein Mitgliedstaat mehr als 96 Sitze erhält; auf Deutschland waren bislang 99 Sitze entfallen. Zu den Ausgangsbedingungen für die Novellierung des EuWG gehörte, drittens, die Entschließung des Europäischen Parlaments vom 22. November 2012 zu den Wahlen zum Europäischen Parlament im Jahr 2014. ${ }^{52}$ Es heißt darin: Das Europäische Parlament „,vertritt angesichts der durch den Vertrag von Lissabon eingeführten neuen Modalitäten für die Wahl der Europäischen Kommission und des sich demzufolge ändernden Verhältnisses zwischen Parlament und Kommission ab den Wahlen 2014 die Ansicht, dass verlässliche Mehrheiten im Parlament für die Stabilität der Legislativverfahren der Union und das reibungslose Funktionieren ihrer Exekutive von entscheidender Bedeutung sein werden, und fordert die Mitgliedstaaten daher auf, in ihrem Wahlrecht gemäß Artikel 3 des Aktes zur Einführung allgemeiner unmittelbarer Wahlen der Abgeordneten der Versammlung geeignete und angemessene Mindestschwellen für die Zuteilung der Sitze festzulegen, um dem in den Wahlen zum Ausdruck gekommenen Wählerwillen gebührend Rechnung zu tragen, bei gleichzeiti-

47 Protokoll über die Rolle der Nationalen Parlamente in der Europäischen Union zum Vertrag von Lissabon, in: Amtsblatt der EU, Nr. C 306 vom 17. Dezember 2007, S. 148-150.

48 Vgl. dazu insgesamt Gabriele Abels/Annegret Eppler (Hrsg.): Auf dem Weg zum Mehrebenenparlamentarismus? Funktionen von Parlamenten im politischen System der EU, Baden-Baden 2011.

49 BVerfGE 123, 267, Leitsatz 2.

50 Matthias Niedobitek: Die Integrationsverantwortung von Bundestag und Bundesrat nach dem „Lissabon-Urteil“ des Bundesverfassungsgerichts, in: Abels/Eppler: Auf dem Weg zum Mehrebenenparlamentarismus?, 2001, S. 159-176, hier Fn. 29.

51 Art. 14 Abs. 2 S. 4 EUV.

52 Europäisches Parlament: Entschließung des Europäischen Parlaments vom 22. November 2012 zu den Wahlen zum Europäischen Parlament im Jahr 2014 (2012/2829 (RSP)), P7_TA(2012)0462. 
ger Wahrung der Funktionalität des Parlaments. “53 Adressaten dieser Entschließung waren neben den EU-Organen die Parlamente und Regierungen der Mitgliedstaaten.

Der Bundestag verabschiedete am 13. Juni 2013 in Dritter Lesung das fünfte Gesetz zur Änderung des EuWG,${ }^{54}$ welches in einem neuen $\S 2$, Abs. 7 eine Dreiprozentsperrklausel in das EuWG einfügt. Der Gesetzentwurf war gemeinsam von den Fraktionen CDU/CSU, SPD, FDP und BÜNDNIS 90/DIE GRÜNEN (also ohne die Fraktion DIE LINKE, die eine Sperrklausel ablehnt) eingebracht worden. ${ }^{55}$ In der Begründung des Gesetzentwurfs wurde die Entschließung des Europäischen Parlaments vom 22. November 2012 mit der an die Mitgliedstaaten gerichteten Aufforderung, Mindestschwellen für die Zuteilung der Sitze festzulegen, ausführlich referiert und damit als ein ganz zentraler Orientierungspunkt für den Bundestag kenntlich gemacht.

Die Einführung einer Dreiprozentsperrklausel wird, mit Blick auf die Entscheidung des Verfassungsgerichts vom November 2011, wie folgt begründet:

Den Gesetzgeber treffe, dem Verfassungsgericht zufolge, eine Beobachtungspflicht, in deren Folge sich für ihn eine abweichende verfassungsrechtliche Beurteilung ergeben könne, wenn sich nämlich die Verhältnisse wesentlich ändern würden. Finde der Wahlgesetzgeber in diesem Sinne veränderte Verhältnisse vor, so müsse er ihnen Rechnung tragen. Maßgeblich für die Frage der weiteren Beibehaltung einer Sperrklausel seien dabei - so das Verfassungsgericht - allein die aktuellen Verhältnisse. Eine solche Veränderung sei gegenüber den Verhältnissen bei der Europawahl 2009, auch seit der Entscheidung des Verfassungsgerichts vom November 2011, eingetreten, die die den Gesetzentwurf einbringenden Fraktionen zu einer abweichenden verfassungsrechtlichen Beurteilung geführt hätten, der sie durch die Einführung einer Dreiprozentklausel Rechnung tragen würden.

Diese Änderung sehen die Fraktionen darin, dass sich eine verfassungsrechtlich erhebliche Entwicklung abzeichne, die - gemäß der Formulierung des Zweiten Senats in seinem Urteil - auf eine stärkere „,antagonistische Profilierung von Regierung und Opposition“56 hinauslaufe. Gemeint sind die größere Bedeutung des Europäischen Parlaments, die Aufstellung von Kandidaten für das Amt des Kommissionspräsidenten durch die europäischen Parteien sowie die damit verbundene Personalisierung und Politisierung der Arbeit des Europäischen Parlaments. Es sei damit zu rechnen, dass eine entsprechend erforderliche Mehrheitsbildung im Europäischen Parlament schwieriger werde, weil nicht von der reibungslosen Fortsetzung der bislang praktizierten Konsensbildung zwischen den beiden großen Fraktionen ausgegangen werden könne.

Es sei im Gegenteil nicht unwahrscheinlich, dass die Wahl des Kommissionspräsidenten stärker zu einer politischen Richtungsentscheidung werde, bei der sich beide großen Fraktionen jeweils um die Bildung einer Mehrheit, unabhängig von der jeweils anderen großen Fraktion, bemühen würden. In der Gesetzesbegründung heißt es daher weiter: „Bei einer starken Zersplitterung der Zusammensetzung des EP steigt dann aber auch das Risiko einer anhaltenden Blockade der parlamentarischen Willensbildung, die zu einer Delegitimierung des Europäischen Parlaments im öffentlichen Ansehen führen könnte. ${ }^{\text {" } 57}$ Dem entgegenzuwirken, werde vom Verfassungsgericht als hinreichende Rechtfertigung für eine Einschrän-

53 Ebenda, Ziffer 4.

54 Fünftes Gesetz zur Änderung des Europawahlgesetzes vom 7. Oktober 2013.

55 Gesetzentwurf der Fraktionen CDU/CSU, SPD, FDP und BÜNDNIS 90/DIE GRÜNEN. Entwurf eines Fünften Gesetzes zur Änderung des Europawahlgesetzes, Bundestagsdrucksache 17/13705.

56 BVerfGE 129, 130, Rn. 109.

57 Gesetzentwurf der Fraktionen CDU/CSU, SPD, FDP und BÜNDNIS 90/DIE GRÜNEN, S. 6. 
kung der Erfolgswertgleichheit der Wahl anerkannt, da mit einer Sperrklausel einer konkret möglichen relevanten Funktionsbeeinträchtigung des Europäischen Parlaments entgegengewirkt würde.

In der zweiten und dritten Beratung des Gesetzentwurfs im Bundestag am 13. Juni $2013^{58}$ wurde von Vertretern der den Entwurf einbringenden Fraktionen unter Verweis auf die in der Begründung des Entwurfs vorgetragenen Argumente übereinstimmend unterstrichen, dass der Bundestag ,die Entscheidung über die Einführung einer Dreiprozentklausel in Ausfüllung des ihm zustehenden Gestaltungsspielraums trifft. " ${ }^{59}$ Wenn und weil das Verfassungsgericht immer wieder klargestellt habe, ,dass es sich gerade in den Kernbereichen der Legislativmacht des Parlaments nicht zum Ersatzgesetzgeber aufschwingen will“ 60 schließe dies ,dann aber mit ein, dass durch eine sehr weitgehende Interpretation unbestimmter Rechtsbegriffe wie der der Funktionsfähigkeit des Parlaments der Beurteilungsspielraum des Bundestages nicht quasi durch die Hintertür praktisch auf null reduziert werden darf.“61 Zur Bedeutung der Sperrklausel wird ganz konkret ausgeführt: „Ohne Sperrklausel kämen aus Deutschland etwa 13 oder 14 Parteien, und zwar - darauf kommt es an - zusätzlich gerade solche, die weder den Parteifamilien angehören, die sich im Europäischen Parlament zu Fraktionen zusammenfinden, noch von diesen integriert werden könnten. Insofern würden wir ohne Sperrklausel eine Zahl von fraktionslosen Parlamentariern produzieren, die das immer wichtigere Funktionieren des Zusammenwirkens der Institutionen gefährden könnten."62

In der Debatte wurde ein weiteres Argument vorgetragen: Die Zersplitterung der deutschen Delegation, mit künftig 96 Parlamentariern, werfe auch ein Repräsentationsproblem auf, „weil es einem einzelnen Abgeordneten eben nicht gelingen kann, die Brüsseler oder Straßburger Entscheidungen gegenüber dem deutschen Wahlbürger zu vertreten. Insofern wird aus diesem machtpolitischen Argument auch ein verfassungsrechtliches. “63

In der Parlamentsdebatte waren auch kritische Anmerkungen zur Entscheidung des Verfassungsgerichts vom November 2011 unüberhörbar: „Das Europäische Parlament entwickelt sich weiter. Die Momentaufnahme, die Karlsruhe 2011 gemacht hat, war schon damals kritisch. Für die Wahl 2014 ist sie falsch; das sage ich ganz deutlich. Die andere Funktionsweise des Parlaments, auch die Europäisierung der Parteien und der Parteienzusammenschlüsse wurde nicht richtig vorausgesehen und nicht richtig eingeschätzt. Deswegen ist es richtig, hier noch einmal diesen Versuch zu machen." ${ }^{64}$ In der Debatte wurde einerseits Zuversicht geäußert, „dass uns im Falle eines Falles [also bei einer erneuten Klage, diesmal gegen die Dreiprozentklausel, Anmerkung des Verfassers] die Karlsruher Richter bestätigen werden, dass wir uns im Rahmen des uns zustehenden Gestaltungsspielraums bewegt haben.“65 Andererseits war aber auch von „Restzweifeln“ die Rede, „ob ein Berichterstatter, der eine Fünfprozenthürde vehement für verfassungswidrig gehalten hat, seine Haltung in einem zukünftigen Urteil revidieren wird."66

58 Deutscher Bundestag. Stenographischer Bericht. 246. Sitzung. Berlin, Donnerstag, den 13. Juni 2013, Plenarprotokoll 17/246, S. 31430 D-31437 C.

59 Reinhard Grindel (CDU/CSU), in: ebenda, S. 31431 A-31432 A, hier S. 31431 C.

60 Grindel, in: ebenda.

61 Grindel, in: ebenda.

62 Grindel, in: ebenda, S. 31432 A.

63 Stefan Ruppert (FDP), in: ebenda, S. 31434 B.

64 Wolfgang Wieland (BÜNDNIS 90/DIE GRÜNEN), in: ebenda, S. 31436 A.

65 Grindel, in: ebenda, S. 31432 A.

66 Ruppert, in: ebenda, S. 31434 C. 
Die Fraktion BÜNDNIS 90/DIE GRÜNEN hatte in den Beratungen über die Gesetzesnovelle im Innenausschuss einen Vorschlag eingebracht, ${ }^{67}$ der auf die Existenz der Parteien auf europäischer Ebene, die ihnen zugeschriebenen Funktionen und ihre Entwicklungsperspektive ausgerichtet war. Danach sollte die Dreiprozentsperrklausel nicht für die Wahlvorschläge der Wahlvorschlagsberechtigten gelten, die Teil einer (nach dem EU-Parteienstatut anerkannten) Partei auf europäischer Ebene sind, ,wenn diese Partei in der Wahl in anderen Mitgliedstaaten der Europäischen Union mindestens 24 Mandate errungen hat und bei Berücksichtigung des Wahlvorschlags diese Partei mit Abgeordneten aus einem Viertel der Mitgliedstaaten der Europäischen Union im Parlament vertreten wäre. “68 Zur Begründung wurde folgendes ausgeführt: Die Dreiprozentsperrklausel solle mit Blick auf das europapolitische Ziel - Stärkung der Handlungsfähigkeit des Europäischen Parlaments - abgemildert und zielgerichtet europäisch ausgerichtet werden. Gemäß der Geschäftsordnung des Europäischen Parlaments bestehe eine Fraktion aus mindestens 25 Abgeordneten aus mindestens einem Viertel der Mitgliedstaaten. „Ziehen daher bereits aus einer hinreichenden Zahl anderer Mitgliedstaaten 24 Abgeordnete der entsprechenden politischen Richtung ein, ist gesichert, dass es nicht zu einer die Handlungsfähigkeit des EP gefährdenden Zersplitterung kommt, weil eine Fraktionsbildung möglich ist. “69 Dieser in der öffentlichen Diskussion wenig beachtete, aber sehr interessante und zweifellos zukunftsorientierte, ${ }^{70}$ zum gegenwärtigen Zeitpunkt aber wohl zu ehrgeizige Änderungsantrag wurde im Innenausschuss mit den Stimmen der Fraktionen CDU/CSU, FDP und DIE LINKE bei Stimmenthaltung der Fraktion der SPD abgelehnt.

\section{Ausblick}

Unmittelbar nach Inkrafttreten des novellierten deutschen EuWG am 7. Oktober 2013 wurden beim Bundesverfassungsgericht Klagen gegen die Dreiprozentsperrklausel eingereicht. Bei den Klägern handelt es sich um die Freien Wähler, die ÖDP, die Piratenpartei, die NPD und die Republikaner. Es ist davon auszugehen, dass das Gericht die Klagen rasch, also rechtzeitig vor den auf den 25. Mai 2014 terminierten Europawahlen in Deutschland behandelt und eine Entscheidung trifft. ${ }^{71}$ Wie die Würdigung des Gerichts ausfällt, ist offen. Entscheidend wird sein, wie das Gericht das zweifellos überzeugende Argument von gegenüber seiner Entscheidung von 2011 geänderten Verhältnissen bewertet. Im Übrigen enthält auch der am 27. November 2013 veröffentlichte Koalitionsvertrag der Großen Koalition von CDU, CSU und SPD eine Aussage, die die vom Bundestag im neuen Europawahlgesetz

67 Dieser im Innenausschuss eingebrachte Änderungsantrag samt Begründung ist auch in der Beschlussempfehlung und dem Bericht des Innenausschusses enthalten. Vgl. Änderungsantrag der Fraktion Bündnis 90/Die Grünen im Ausschuss. Entwurf eines Fünften Gesetzes zu Änderung des Europawahlgesetzes, Ausschussdrucksache 17(4)761; Beschlussempfehlung und Bericht des Innenausschusses (4. Ausschuss) zu dem Gesetzentwurf der Fraktionen CDU/CSU, SPD, FDP und BÜNDNIS 90/DIE GRÜNEN - Drucksache 17/13705 - Entwurf eines Fünften Gesetzes zur Änderung des Europawahlgesetzes, Bundestagsdrucksache 17/13935, S. 4.

68 Änderungsantrag der Fraktion Bündnis 90/Die Grünen im Ausschuss, 2013.

69 Ebenda.

70 Bereits anlässlich der Beratungen zum deutschen Europawahlgesetz für die ersten Direktwahlen 1979 waren entsprechende Überlegungen angestellt worden. Wäre eine solche Ausnahme von der (Fünfprozent-)Sperrklausel seinerzeit vorgesehen worden, hätte das bedeutet, dass Kandidaten der FDP, die bei drei Europawahlen an der Hürde gescheitert war, gleichwohl als Mitglieder der Fraktion der Liberalen ins Europäische Parlament hätten einziehen können. Näheres dazu bei Rudolf Hrbek: Das Deutsche Wahlgesetz zum Europäischen Parlament in der Parteienkontroverse, in: Zeitschrift für Parlamentsfragen 2/1978, S. 168-179.

71 Die mündliche Verhandlung ist auf den 18. Dezember 2013 terminiert. 
beschlossene Regelung sowie die vom Europäischen Parlament im November 2012 erhobene Forderung unterstützt. ${ }^{72}$

72 Der Koalitionsvertrag enthält in seinem Abschnitt 6 („Starkes Europa. Europapolitische Verantwortung Deutschlands.“) folgenden Passus: „Die Bundesregierung unterstützt die Einrichtung eines einheitlichen europäischen Wahlrechts, um verlässliche Mehrheiten im Europäischen Parlament für die Legislativverfahren der Union sicherzustellen. In diesem Zusammenhang sollte eine angemessene Mindestschwelle für die Zuteilung der Sitze festgelegt werden“. 